\title{
Thesaurus-Based Search in Large Heterogeneous Collections
}

\author{
Jan Wielemaker ${ }^{1}$, Michiel Hildebrand ${ }^{2}$, \\ Jacco van Ossenbruggen ${ }^{2}$, and Guus Schreiber ${ }^{3}$ \\ ${ }^{1}$ University of Amsterdam, Human Computer Studies (HCS), The Netherlands \\ ${ }^{2}$ CWI Amsterdam, The Netherlands \\ ${ }^{3}$ VU University Amsterdam, The Netherlands
}

\begin{abstract}
In cultural heritage, large virtual collections are coming into existence. Such collections contain heterogeneous sets of metadata and vocabulary concepts, originating from multiple sources. In the context of the E-Culture demonstrator we have shown earlier that such virtual collections can be effectively explored with keyword search and semantic clustering. In this paper we describe the design rationale of ClioPatria, an open-source system which provides APIs for scalable semantic graph search. The use of ClioPatria's search strategies is illustrated with a realistic use case: searching for "Picasso". We discuss details of scalable graph search, the required OWL reasoning functionalities and show why SPARQL queries are insufficient for solving the search problem.
\end{abstract}

\section{Introduction}

Traditionally, cultural heritage, image and video collections use proprietary database systems and often their own thesauri and controlled vocabularies to index their collection. Many institutions have made or are making (parts of) their collections available online. Once on the web, each institution, typically, provides access to their own collection. The cultural heritage community now has the ambition to integrate these isolated collections and create a potential source for many new inter-collection relationships. New relations may emerge between objects from different collections, through shared metadata or through relations between the thesauri.

The MultimediaN E-culture project 11 explores the usability of semantic web technology to integrate and access museum data in a way that is comparable to the MuseumFinland project 11. We focus on providing two types of end-user functionality on top of heterogeneous data with weak domain semantics. First, keyword search, as it has become the de-facto standard to access data on the web. Secondly, thesaurus-based annotation for professionals as well as amateurs.

This document is organised as follows. In Sect. 2 we first take a closer look at our data and describe our requirements by means of a use case. In section Sect. 3 we take a closer look a search and what components are required to

\footnotetext{
1 http://e-culture.multimedian.nl
} 
realise keyword search in a large RDF graph. The ClioPatria infrastructure is described in section Sect. 4, together with some illustrations on how ClioPatria can be used. We conclude the paper with a discussion where we position our work in the Semantic Web community.

\section{Materials and Use Cases}

Metadata and vocabularies. In our case study we collected descriptions of 200,000 objects from six collections annotated with six established thesauri and several proprietary controlled keyword lists, which adds up to 20 million triples. We assume this material is representative for the described domain. Using semantic web technology, it is possible to unify the data while preserving its richness. The procedure is described elsewhere [2] and summarised here 2

The MultimediaN E-Culture demonstrator harvests metadata and vocabularies, but assumes the collection owner provides a link to the actual data object, typically an image of a work such as a painting, a sculpture or a book. When integrating a new collection into the demonstrator we typically receive one or more XML/database dumps containing the metadata and vocabularies of the collection. Thesauri are translated into RDF/OWL, where appropriate with the help of the W3C SKOS format for publishing vocabularies 3. The metadata is transformed in a merely syntactic fashion to RDF/OWL triples, thus preserving the original structure and terminology. Next, the metadata schema is mapped to VRA3, a specialisation of Dublin Core for visual resources. This mapping is realized using the 'dumb-down' principle by means of rdfs:subPropertyOf and rdfs:subClass0f relations. Subsequently, the metadata goes through an enrichment process in which we process plain-text metadata fields to find matching concepts from thesauri already in the demonstrator. For example, if the dc:creator field contains the string Pablo Picasso, than we will add the concept ulan:500009666 from ULAN to the metadata. Most enrichment concerns named entities (people, places) and materials. Finally, the thesauri are aligned using owl:sameAs and skos:exactMatch relations. For example, the art style Edo from a local ethnographic collection was mapped to the same art style in AAT5 (see the use cases for an example why such mappings are useful). Our current database (April 2008) contains 38,508 owl:sameAs and 9,635 skos:exactMatch triples and these numbers are growing rapidly.

After this harvesting process we have a graph representing a connected network of works and thesaurus lemmas that provide background knowledge. VRA and SKOS provide - weak - structure and semantics. Underneath, the richness of the original data is still preserved. The data contains many relations that are not covered by VRA or SKOS, such as relations between artists (e.g. ULAN teacherOf relations) and between artists and art styles (e.g. relations between

\footnotetext{
${ }^{2}$ The software can be found at http://sourceforge.net/projects/annocultor

${ }^{3}$ Visual Resource Association, http://www.vraweb.org/projects/vracore4/

${ }^{4}$ Union List of Artist Names is a thesaurus of the Getty foundation.

${ }^{5}$ Art \& Architecture Thesaurus, another Getty thesaurus.
} 


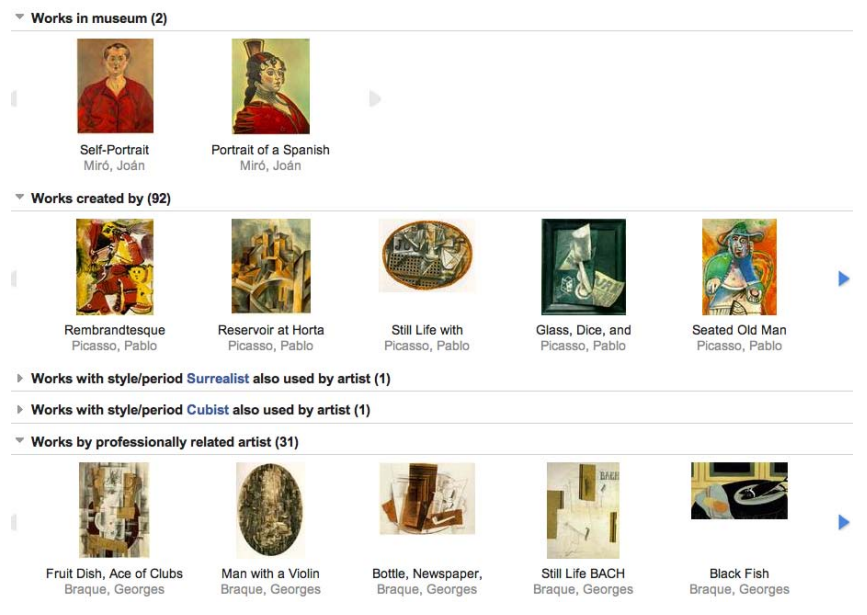

Fig. 1. Clustered result searching "picasso"

AAT art styles and ULAN artists [4). These relations are covered by their original schema. Their diversity and lack of defined semantics make it hard to map them to existing ontologies and provide reasoning based on this mapping.

Use cases. Assume a user is typing in the query "picasso". Despite the name Picasso is a reasonably unique in the art world, the user may still have many different intentions with this simple query: a painting by Picasso, a painting of Picasso, the styles Picasso has worked in? Without an elaborate disambiguation process it is impossible to tell in advance.

Fig. 1] show part of the results of this query in the MultimediaN demonstrator. We see several clusters of search results. The first cluster contains works from the Picasso Museum, the second cluster contains works by Pablo Picasso (only first five hits shown; clicking on the arrow allows the user to inspect all results); clusters of surrealist and cubist paintings (styles that Picasso worked in; not shown for space reasons), and works by George Braque (a prominent fellow Cubist painter, but the works shown are not necessarily cubist). Other clusters include works made from picasso marble and works with Picasso in the title (includes two self portraits). The basic idea is that we are aiming to create clusters of related objects such that the user can afterwards choose herself what she is interested in. We have found that even in relatively small collections of $100 \mathrm{~K}$ objects, users discover interesting results they did not expect. We have termed this type of search tentatively 'post-query disambiguation': in response to a simple keyword query the user gets (in contrast to, for example, Google image search) semantically-grouped results that enable further detailing of the query. It should be pointed out that the knowledge richness of the cultural heritage domain allows this approach to work. In less rich domains such an approach is less likely to provide added value. Notably typed resources and relations give meaning to the path linking a literal to a target object. 


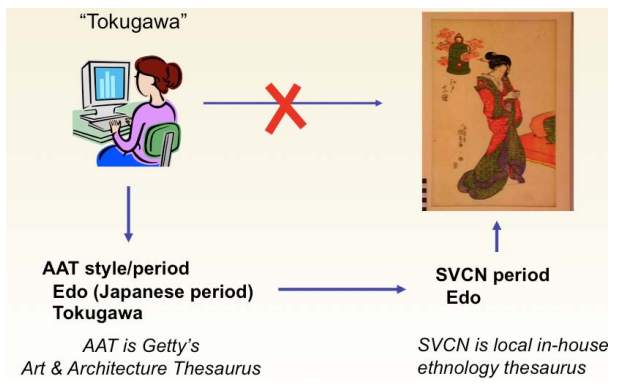

Fig. 2. Explore alignment to find Edo painting from "tokugawa"

Another typical use case for search concerns the exploitation of vocabulary alignments. The Holy Grail of the unified cultural-heritage thesaurus does not exist and many collection owners have their own home-grown variants. Consider the situation in Fig. 2, which is based on real-life data. A user is searching for "tokugawa". This Japanese term has actually two major meanings in the heritage domain: it is the name of a 19th century shogun and it is a synonym for the Edo style period. Assume for a moment that the user is interested in finding works of the latter type. The Dutch ethnographic museum in Leiden actually has works in this style in its digital collection, such as the work shown in the topright corner. However, the Dutch ethnographic thesaurus SVCN, which is being used by the museum for indexing purposes, only contains the label "Edo" for this style. Fortunately, another thesaurus in our collection, the aforementioned AAT, does contain the same concept with the alternative label "Tokugawa". In the harvesting process we learned this equivalence link (quite straightforward: both are Japanese styles with matching preferred labels). The objective of our graph search is to enable to make such matches.

Although this is actually an almost trivial alignment, it is still extremely useful. The cultural-heritage world (like any knowledge rich domain) is full of such small local terminology differences. Multilingual differences should also be taken into consideration here. If semantic-web technologies can help making such matches, there is a definite added value for users.

\section{Required Methods and Components}

In this section we study the methods and components we need to realise the keyword search described above. Our experiments indicate that meaningful matches between keyword and target often involve chains up to about five relations. At this distance there is a potentially huge set of possible targets. The targets can be organised by rating based on semantics or statistics and by clustering based on the graph pattern linking a literal to the target. We discuss three possible approaches: querying using a fixed set of graph patterns, completely unconstrained graph search and best-first exploration of the graph. 
Using a set of fixed queries. A cluster as shown in Fig. 1 is naturally represented as a graph pattern as found in many semantic web query languages. If we can enumerate all possible meaningful patterns of properties that link literals to targets, we reduce the search process to finding instances of all these graph patterns. This would be a typical approach in Semantic Web applications such as DBin [5]. This approach is, however, not feasible for highly heterogenous data sets. Our current data contains over 600 properties, most of which do not have a very well defined meaning (e.g. detailof, cooperatedWith, usesStyle). If we combine this with our observation that is it quite common to find valuable results at 4 or even 5 steps from the initial keywords, we have to evaluate a very large number of possible patterns. To a domain expert, it is obvious that the combination of cooperatedWith and hasstyle can be meaningful while " $A$ died in $P$, where $B$ was born" is generally meaningless, but the set of possible combinations to consider is very large. Automatic rating of this type of relation pattern is, as far as we know, not feasible. Even if the above is possible, new collections and vocabularies often come with new properties, which must all be considered in combination to the already created patterns.

Using graph exploration. Another approach is to explore the graph, looking for targets that have, often indirectly, a property with matching literal. This implies we search the graph from Object to Subject over arbitrary properties, including triples entailed by owl:inverse0f and owl: SymmetricProperty. We examine the scalability issues using unconstrained graph patterns, after which we examine an iterative approach.

Considering a triple store that provides reasoning over owl:inverse0f and owl: SymmetricProperty it is easy to express an arbitrary path from a literal to a target object with a fixed length. The total result set can be expressed as a union of all patterns of fixed length up to (say) distance 5. Table 1 provides the statistics for some typical keywords at distances 3 and 5 . The table shows total visited and unique results for both visited nodes and targets found which indicates that the graph contains a large number of alternative paths and the implementation must deal with these during the graph exploration to reduce the amount of work. Even without considering the required post-processing to rank and cluster the results it is clear that we cannot obtain interactive response times (of at most a few seconds) using this approach.

Fortunately, a query system that aims at human users only needs to produce the most promising results. This can be achieved by introducing a distance measure and doing best-first search until our resources are exhausted (anytime algorithm) or we have a sufficient number of results. The details of the distance measure are still subject of research [6, but not considered vital to the architectural arguments in this article. The complete search and clustering algorithm is given in Fig. 3. In our experience, the main loop requires about 1,000 iterations to obtain a reasonable set of results, which leads to acceptable performance when the loop is pushed down to the triple store layer. 
Table 1. Statistics for exploring the search graph for exactly Distance steps (triples) from a set of literals matching Keyword. Literals is the number of literals holding a word with the same stem as Keyword; Nodes is the number of nodes explored and Targets is the number of target objects found. Time is on an Intel Core duo X6800.

\begin{tabular}{|c|c|c|c|c|c|c|c|}
\hline Keyword & $\overline{\text { Distance }]}$ & terals & $\begin{array}{r}\text { Nod } \\
\text { Visited }\end{array}$ & $\begin{array}{l}\text { es } \\
\text { Unique }\end{array}$ & $\begin{array}{r}\text { Targ } \\
\text { Visited }\end{array}$ & $\begin{array}{l}\text { ets } \\
\text { Unique }\end{array}$ & $\begin{array}{l}\text { Time } \\
\text { (sec.) }\end{array}$ \\
\hline tokugawa & 3 & 21 & 1,346 & $\overline{1,228}$ & 913 & 898 & $\overline{0.02}$ \\
\hline steen & 3 & 1,070 & 21,974 & 7,897 & 11,305 & 3,658 & 0.59 \\
\hline picasso & 3 & 85 & 9,703 & 2,399 & 2,626 & 464 & 0.26 \\
\hline rembrandt & 3 & 720 & 189,611 & 9,501 & 141,929 & 4,292 & 3.83 \\
\hline impressionism & 3 & 45 & 7,142 & 2,573 & 3,003 & 1,047 & 0.13 \\
\hline amsterdam & 3 & 6,853 & $1,327,797$ & 421,304 & 681,055 & 142,723 & 39.77 \\
\hline tokugawa & 5 & 21 & 11,382 & 2,432 & 7,407 & 995 & 0.42 \\
\hline steen & 5 & 1,070 & $1,068,045$ & 54,355 & 645,779 & 32,418 & 19.42 \\
\hline picasso & 5 & 85 & 919,231 & 34,060 & 228,019 & 6,911 & 18.76 \\
\hline rembrandt & 5 & 720 & $16,644,356$ & 65,508 & $12,433,448$ & 34,941 & 261.39 \\
\hline impressionism & 5 & 45 & 868,941 & 50,208 & 256,587 & 11,668 & 18.50 \\
\hline amsterdam & 5 & 6,853 & $37,578,731$ & 512,027 & $23,817,630$ & 164,763 & 620.82 \\
\hline
\end{tabular}

Term search. The combination of best-first graph exploration with semantic clustering, as described above, works well for 'post-query' disambiguation of results in exploratory search tasks. It is, however, less suited for quickly selecting a known thesaurus term. The latter is often needed in semantic annotation and 'pre-query' disambiguation search tasks. For such tasks we rely on the proven autocompletion technique, which allows us to quickly find resources related to the prefix of a label or a word inside a label, organise the results (e.g. organise cities by country) and provide sufficient context (e.g. date of birth and death of a person). Often results can be limited to a sub-hierarchy of a thesaurus, expressed as an extra constraint using the transitive skos:broader property. Although the exact technique differs, the technical requirements to realise this type of search is similar to the keyword search described above.

Literal matching. Similar to document retrieval, we start our search from a rated list of literals that contain words with the same stem as the searched keyword. Unlike document retrieval systems such as Swoogle [7] or Sindice [8, we are not interested in which RDF documents contain the matching literals, but which semantically related target concepts are connected to them. Note that term search as described above requires finding literals from the prefix of a contained word that is sufficiently fast to be usable in autocompletion interfaces (see also [9]).

Using SPARQL. If possible, we would like our search software to connect to an arbitrary SPARQL endpoint. Considering the fixed query approach, each pattern is naturally mapped onto a SPARQL graph pattern. Unconstrained graph search is easily expressed too. Expressed as a CONSTRUCT query, the query engine can return a minimal graph without duplicate paths.

Unfortunately, both approaches proved to be infeasible implementation strategies. The best-first graph exploration requires one (trivial) SPARQL query to 
1. Find literals that contain the same stem as the keywords, rate them on minimal edit distance (short literal) or frequency (long literal) and sort them on the rating to form the initial agenda

2. Until satisfied or empty agenda, do

(a) Take highest ranked value from agenda as $O$. Find $\operatorname{rdf}(S, P, O)$ terms. Rank the found $S$ on the ranking of $O$, depending on $P$. If $P$ is a subProperty of owl:sameAs, the ranking of $S$ is the same as $O$. If $S$ is already in the result set, combine their values using $R=1-\left(\left(1-R_{1}\right) \times\left(1-R_{2}\right)\right)$. If $S$ is new, insert it into agenda, else reschedule it in the agenda.

(b) If $S$ is a target, add it to the targets. Note that we must consider $\operatorname{rdf}(O, I P, S)$ if there is an inverse $\mathbf{O f}(P, I P)$ or $P$ is symmetric.

3. Prune resulting graph from branches that do not end in a target.

4. Smush resources linked by owl:sameAs, keeping the most linked resource.

5. Cluster the results

(a) Abstract all properties to their VRA or SKOS root property (if possible).

(b) Abstract resources to their class, except for instances of skos: Concept and the top-10 ranked instances.

(c) Place all triples in the abstract graph. Form (RDF) Bags of resources that match to an abstracted resource and use the lowest common ancestor for multiple properties linking two bags of resources.

6. Complete the nodes in the graph with label information for proper presentation.

Fig. 3. Best first graph search and clustering algorithm

find the neighbours of the next node in the agenda for each iteration to update the agenda and to decide on the next node to explore. Latency and volume of data transfer make this infeasible when using a remote triple store.

The reasoning for clustering based on the property hierarchy cannot be expressed in SPARQL, but given the size and stability of the property hierarchy we can transfer the entire hierarchy to the client and use local reasoning. After obtaining the clustered results, the results need to be enriched with domain specific key information (title and creator) before they can be presented to the user. Requesting the same information from a large collection of resources can be realised using a rather inelegant query as illustrated below.

SELECT ?11 ?12 ...

WHERE $\{\{$ ulan:artists1 rdfs:label ?11\} UNION

\{ ulan:artists2 rdfs:label ?12 \} UNION

$\cdots$

Regular expression literal matching cannot support match on stem. Prefix and case insensitive search for contained word can be expressed. Ignoring diacritic marks during matching as generally needed for multi-script matching is not supported by the SPARQL regular expression syntax.

We conclude that remote access is inadequate for adaptive graph exploration and SPARQL is incapable of expressing lowest common parent problems and relevant literal operations and impractical for enriching computed result sets. 


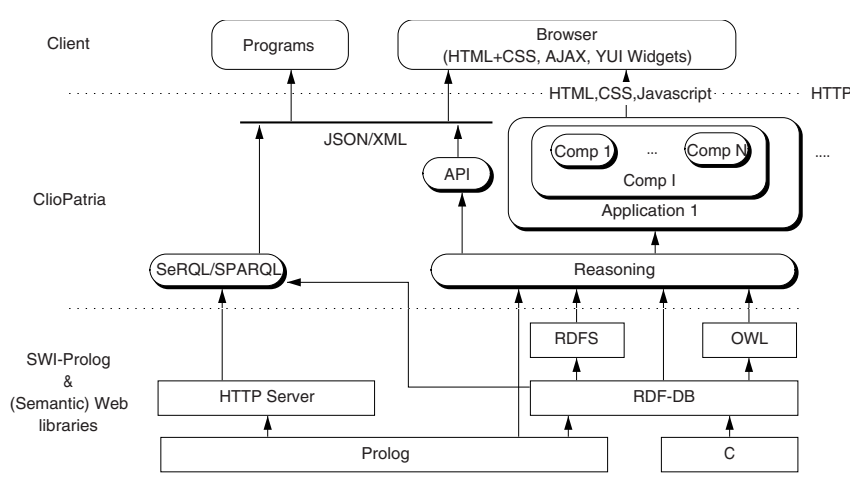

Fig. 4. Overall architecture of the ClioPatria server

\section{Summary of requirements for search.}

- Obtain rated list of literals from stem and prefix of contained words.

- Entailment over owl:inverse0f and owl:SymmetricProperty.

- Entailment over owl:TransitiveProperty to limit the domain of term search.

- Entailment over owl:sameAs for term search.

- Graph exploration requires tight connection to the triple store.

- Reasoning with types as well as the class, concept and property hierarchy. This includes finding the lowest common parent of a set of resources.

\section{The ClioPatria Search and Annotation Toolkit}

We have realised the functionality described in the previous section on top of the SWI-Prolog6 web and semantic web libraries [1011]. This platform provides a scalable in-core RDF triple store [12] and a multi-threaded HTTP server library. ClioPatria 7 is the name of the reusable core of the E-culture demonstrator, the architecture of which is illustrated in Fig. 4. First, we summarise some of the main features of ClioPatria.

- Running on a Intel core duo X6800@2.93GHz, 8GB, 64-bit Linux it takes 120 seconds elapsed time to load the 20 million triples. The server requires $4.3 \mathrm{~Gb}$ memory for 20 million triples $(2.3 \mathrm{~Gb}$ in 32 -bit mode). Time and space requirements grow practically linear in the amount of triples.

- The store provides safe persistency and maintenance of provenance and change history based on a (documented) proprietary file format.

- Deleting and modifying triples complicates maintenance of the pre-computed entailment. Therefore, reasoning is as much as possible based on backward chaining, which fits with Prolog's search resolution.

6 http://www.swi-prolog.org

7 Open source from http://e-culture.multimedian.nl/software.html 


\subsection{Client-Server Architecture}

In contrast to client-only architectures such as Simile's Exhibit [13, ClioPatria has a client-server architecture. The core functionality is provided as HTTP APIs by the server. The results are served as presentation neutral data objects using the JSON 8 serialization and can thus be combined with different presentation and interaction strategies. Within ClioPatria, the APIs are used by its web applications. In addition, the APIs can be used by third party applications to create mashups. The ClioPatria toolkit contains web applications for search and annotation. The end-user applications are a combination of server side generated HTML and client side JavaScript interface widgets.

In the MultimediaN E-Culture demonstrator 9 ClioPatria's web application for search and annotation are used. The K-Space European Network of Excellence is using ClioPatria to search new 10 . At the time of writing Europeand 11 is setting up ClioPatria as a demonstrator to provide multilingual access to a large collection of very divers cultural heritage data. The ClioPatria API provided by the E-Culture Project is also used by the CATCH/CHIP project Tour Wizard that won the 3rd prize at the Semantic Web Challenge of 2007. For the semantic search functionality CHIP uses the web services provided by the ClioPatria API.

\subsection{Output Formats}

Server side we have two types of presentation oriented output routines. Components are Prolog grammar rules that define reusable parts of a page. Components can embed each other. Applications produce an entire HTML page that largely consists of configured components. Applications automatically add the required CSS and JavaScript based on dependency declarations.

Client side presentation and interaction is built on top of YUI JavaScript widget library 12 ClioPatria contains widgets for autocompletion, a search result viewer, a detailed view on a single resource, and widgets for semantic annotation fields. The result viewer can visualise data in thumbnail clusters, a geographical map, Simile Exhibit, Simile Timeline and a Graphvit 13 graph visualisation.

\subsection{APIs}

ClioPatria provides programmatic access to the RDF data through HTTP. http://e-culture.multimedian.nl/demo/doc/ The query API provides standardized access to the data via the SeRQL and SPARQL. As we have shown in Sect. 3 such a standard query API is not sufficient to provide the intended keyword search functionality. Therefore, ClioPatria provides an additional search API for

\footnotetext{
8 http://www.json.org

9 http://e-culture.multimedian.nl/demo/search

10 http://newsml.cwi.nl/explore/search

11 http://www.europeana.eu/

12 http://developer.yahoo.com/yui/

13 http://www.graphviz.org/
} 
keyword-based access to the RDF data. In addition, ClioPatria provides APIs to get resource-specific information, update the triple store and cache media items. This paper only discusses the query and search API in more detail.

Query API. The SeRQL/SPARQL library provides a semantic web query interface that is compatible to Sesame 14 and provides open and standardised access to the RDF data stored in ClioPatria. Both SeRQL and SPARQL are translated into a Prolog query that relies on the $\mathbf{r d f}(S, P, O)$ predicate provided by the RDF store and on auxiliary predicates that realise functions and filters defined by SeRQL and SPARQL. Conjunctions of rdf/3 statements and filter expressions are optimised through reordering based on statistical information provided by the store 15 .

Search API. The search API provides services for graph search (Fig. 3) and term search (Sect. 3). Both services return their result as a JSON object (using the serialisation for SPARQL SELECT queries [16]). Both services can be configured with several parameters. General search API parameters are:

- query(string $\mid U R I)$ : the search query.

- filter(false|Filter): constrains the results to match a combination of Filter primitives, typically OWL class descriptions that limit the results to instances that satisfy these descriptions. Additional syntax restricts results to resources used as values of properties of instances of a specific class.

- groupBy(false $\mid$ path $\mid$ Property): if path, cluster results by the abstracted path linking query to target. If a property is given, group the result by the value on the given property.

- $\operatorname{sort}($ path_length $\mid$ score $\mid$ Property): Sort the results on path-length, semantic distance or the value of Property.

- info(false | PropertyList): augment the result with the given properties and their values. Examples are skos:prefLabel, foaf:depicts and dc:creator.

- view(thumbnail|map|timeline|graph|exhibit): shorthands for specific property lists of info.

- sameas(Boolean): smushes equivalent resources, as defined by owl: sameAs or skos: exactMatch into a single resource.

Consider the use case discussed in Sect. 2. Clustered results that are semantically related to keyword "picasso" can be retrieved through the graph search API with this HTTP request:

/api/search?query=picasso\&filter=vra: Work\&groupBy=path\&view=thumbnail

Parameters specific to the graph search API are:

- abstract(Boolean): enables the abstraction of the graph search paths over rdfs:subClass0f and rdfs:subProperty0f, reducing the number of clusters.

- bagify(Boolean): puts (abstracted) resources of the same class with the same (abstracted) relations to the rest of the graph in an RDF bag. I.e. convert a set of triples linking a painter over various sub properties of dc:creator to multiple instances of vra:Work, into an RDF bag of works and a single triple linking the painter as dc:creator to this bag. 
- steps(Integer): limits graph exploration to expand less than Integer nodes.

- threshold(0.0.1.0): cuts off the graph exploration on semantic distance.

For annotation we can use the term search API to suggest terms for a particular annotation field. For example, suppose a user has typed the prefix "pari" in a location annotation field that only allows European locations. We can request matching suggestions by using the URI below, filtering the results to resources that can be reached from tgn:Europe using skos:broader transitively:

/api/autocomplete?query=pari\&match=prefix\&sort=rdf $\mathrm{s}:$ label\& filter=\{"reachable": \{"relation": "skos:broader", "value" : "tgn:Europe"\}\}

Parameters specific to the term search API are:

- match(prefix|stem|exact): defines how the syntactic matching of literals is performed. Autocompletion, for example, requires prefix match.

- $\operatorname{property}($ Property, 0.0.1.0): is a list of RDF property-score pairs which define the values that are used for literal matching. The score indicates preference of the used literal in case a URI is found by multiple labels. Typically preferred labels are chosen before alternative labels.

- preferred(skos:inScheme, URI): if URIs are smushed the information of the URI from the preferred thesaurus is used for augmentation and organisation.

- compound(Boolean): if true, filter results to those where the query matches the information returned by the info parameter. For example, a compound query paris, texas can be matched in two parts against a) the label of the place Paris and b) the label of the state in which Paris is located.

\section{Discussion and Conclusion}

In this paper we analysed the requirements for searching in large, heterogeneous collections with rich, but formally ill-defined semantics. We presented the ClioPatria software architecture we used to explore this topic. Three characteristics of ClioPatria have proved to be a frequent source of discussion: the non-standard API, the central in-core triple store model and the lack of full OWL DL support.

API standardisation. First, ClioPatria's architecture is based on various client-side JavaScript Web applications around a server-side Prolog-based reasoning engine and triple store. As discussed in this paper, the server functionality required by the Web clients extends that of an off-the-shelf SPARQL endpoint. This makes it hard for Semantic Web developers of other projects to deploy our Web applications on top of their own SPARQL-based triple stores. We acknowledge the need for standardized APIs in this area. We hope that the requirements discussed in this paper provide a good starting point to develop the next generation Semantic Web APIs that go beyond the traditional database-like query functionality currently supported by SPARQL.

Central, in-core storage model. From a data-storage perspective, the current ClioPatria architecture assumes images and other annotated resources to 
reside on the Web. All metadata being searched, however, is assumed to reside in-core in a central, server-side triple store. We are currently using this setup with a $20 \mathrm{M}$ triples dataset, and are confident our current approach will easily scale up to $150 \mathrm{M}$ triples on modern hardware (32Gb core). Our central in-core model will not scale, however, to the multi-billion triple sets supported by other state-of-the-art triple stores. For future work, we are planning to investigate to what extent we can move to disk-based or, given the distributed nature of the organisations in our domain, distributed storage strategies without giving up the key search functionalities of our current implementation. Distribution of the entire RDF graph is non-trivial. For example, in the keyword search, the paths in the RDF graph from the matching literals to the target resources tend to be unpredictable, varying highly with the types of the resources associated with the matching literals and the type of the target resources. Implementing a fast, semirandom graph walk in a distributed fashion will likely be a significant challenge. As another example, interface components such as a Web-based autocompletion Widget are based on the assumption that a client Web-application may request autocompletion suggestions from a single server, with response times in the 200ms range. Realizing sufficiently fast responses from this server without the server having a local index of all literals that are potential suggestion candidates will also be challenging. Distributing carefully selected parts of the RDF graph, however, could be a more promising option. In our current datasets for example, the subgraphs with geographical information are both huge and connected to the rest of the graph in a limited and predictable fashion. Shipping such graphs to dedicated servers might be doable with only minor modifications to the search algorithms performed by the main server.

Lack of OWL reasoning. From a reasoning perspective, ClioPatria does not provide traditional OWL DL support. First of all, the heterogeneous and open nature of our metadata repositories ensures that even when the individual data files loaded are in OWL DL, their combination will most likely not be. Typical DL violations in this domain are properties being used as a data property with name strings in one collection, and as an object property with URIs pointing to a biographical thesaurus such as ULAN in the other; or rdfs:label properties being used as an annotation property in the schema of one collection and as a data property on the instances of another collection. We believe that OWL DL is a powerful and expressive subset of OWL for closed domains where all data is controlled by a single organisation. It has proved, however, to be unrealistic to use OWL DL for our open, heterogenous Semantic Web application where multiple organisations can independently contribute to the data set.

Secondly, our application requirements require the triple store to be able to flexibly turn on and off certain types of OWL reasoning on a per-query basis. For example, there are multiple URIs in our dataset, from different data sources, representing the Dutch painter Rembrandt van Rijn. Ideally, our vocabulary mapping tools have detected this and have all these URIs mapped to one another using owl:sameAs. For an end-user interested in viewing all information available on Rembrandt, it is likely beneficial to have the system perform owl: sameAs 
reasoning and present all information related to Rembrandt in a single interface, smushing all different URIs onto one. However, an expert end-user annotating an artwork being painted by Rembrandt will, when selecting the corresponding entry from a biographical thesaurus, be interested into which vocabulary source the URI of the selected concept is pointing, and will also be interested in the other vocabularies define entries about Rembrandt, and how the different entries differ. This requires the system to largely ignore the traditional owl:sameAs semantics, present all triples associated with the different URIs separately, along with the associated provenance information. This type of ad-hoc turning on and off of specific OWL reasoning is not supported by most off-the-shelf SPARQL endpoints, but crucial in all realistic multi-thesauri semantic web applications.

Thirdly, we found that our application requirements seldomly rely on extensive subsumption or other typical OWL reasoning. In the weighted graph exploration we basically only consider the graph structure and ignore most of the underlying semantics, with only a few notable exceptions. Results are improved by assigning equivalence relations such as owl: sameAs and skos: exactMatch the highest weight of 1.0. We search the graph in only one direction, the exception being properties being declared as an owl:SymmetricProperty. In case of properties having an owl:inverse0f, we traverse the graph as we would have if all "virtual" inverse triples were materialised. Finally, we use a simple form of subsumption reasoning over the property and class hierarchy when presenting results to abstract from the many small differences in the schemas underlying the different search results.

Conclusion. Our conclusion is that knowledge rich domains such as cultural heritage fit well with Semantic Web technology. This is because of a) the clear practical needs this domain has for integrating information from heterogeneous sources, and b) its long tradition with semantic annotations using controlled vocabularies and thesauri. We strongly feel that studying the real application needs of users working in such domains in terms of their search and reasoning requirements will move ahead the state of the art in Semantic Web research significantly.

\section{References}

1. Hyvönen, E., Junnila, M., Kettula, S., Mäkelä, E., Saarela, S., Salminen, M., Syreeni, A., Valo, A., Viljanen, K.: MuseumFinland - Finnish museums on the semantic web. Journal of Web Semantics 3, 224-241 (2005)

2. Tordai, A., Omelayenko, B., Schreiber, G.: Semantic excavation of the city of books. In: Proc. Semantic Authoring, Annotation and Knowledge Markup Workshop (SAAKM 2007), CEUR-WS, vol. 289, pp. 39-46 (2007), http://ceur-ws.org/Vol-289

3. Miles, A., Bechhofer, S.: SKOS simple knowledge organization system reference. W3C working draft, World-Wide Web Consortium, Latest version (2008), http://www.w3.org/TR/skos-reference

4. de Boer, V., van Someren, M., Wielinga, B.J.: A redundancy-based method for the extraction of relation instances from the web. International Journal of HumanComputer Studies 65, 816-831 (2007) 
5. Tummarello, G., Morbidoni, C., Nucci, M.: Enabling Semantic Web communities with DBin: an overview. In: Cruz, I., Decker, S., Allemang, D., Preist, C., Schwabe, D., Mika, P., Uschold, M., Aroyo, L.M. (eds.) ISWC 2006. LNCS, vol. 4273, Springer, Heidelberg (2006)

6. Rocha, C., Schwabe, D., de Aragao, M.: A hybrid approach for searching in the semantic web. In: Proceedings of the 13th International World Wide Web Conference, New York, USA, pp. 374-383 (2004)

7. Ding, L., Finin, T., Joshi, A., Pan, R., Cost, R.S., Peng, Y., Reddivari, P., Doshi, V.C., Sachs, J.: Swoogle: A Search and Metadata Engine for the Semantic Web. In: Proceedings of the Thirteenth ACM Conference on Information and Knowledge Management, Washington, D.C., USA, pp. 652-659 (2004)

8. Tummarello, G., Oren, E., Delbru, R.: Sindice.com: Weaving the open linked data. In: Aberer, K., Choi, K.-S., Noy, N., Allemang, D., Lee, K.-I., Nixon, L., Golbeck, J., Mika, P., Maynard, D., Mizoguchi, R., Schreiber, G., Cudré-Mauroux, P. (eds.) ASWC 2007 and ISWC 2007. LNCS, vol. 4825, pp. 547-560. Springer, Heidelberg (2007)

9. Bast, H., Weber, I.: The CompleteSearch Engine: Interactive, Efficient, and towards IR\&DB Integration. In: CIDR 2007, Third Biennial Conference on Innovative Data Systems Research, Asilomar, CA, USA, pp. 88-95 (2007)

10. Wielemaker, J., Huang, Z., van der Meij, L.: SWI-Prolog and the web. Theory and Practice of Logic Programming 8, 363-392 (2008) (accepted for publication)

11. Wielemaker, J., Hildebrand, M., van Ossenbruggen, J.: Using Prolog as the fundament for applications on the semantic web. In: Heymans, S., et al. (eds.) Proceedings of ALPSWS 2007, pp. 84-98 (2007)

12. Wielemaker, J., Schreiber, G., Wielinga, B.: Prolog-based infrastructure for RDF: performance and scalability. In: Fensel, D., Sycara, K.P., Mylopoulos, J. (eds.) ISWC 2003. LNCS, vol. 2870, pp. 644-658. Springer, Heidelberg (2003)

13. Huynh, D., Karger, D., Miller, R.: Exhibit: Lightweight structured data publishing. In: 16th International World Wide Web Conference, Banff, Alberta, Canada, ACM Press, New York (2007)

14. Broekstra, J., Kampman, A., van Harmelen, F.: Sesame: A generic architecture for storing and querying rdf and rdf schema. In: Horrocks, I., Hendler, J. (eds.) ISWC 2002. LNCS, vol. 2342, pp. 54-68. Springer, Heidelberg (2002)

15. Wielemaker, J.: An optimised semantic web query language implementation in prolog. In: Gabbrielli, M., Gupta, G. (eds.) ICLP 2005. LNCS, vol. 3668, pp. 128142. Springer, Heidelberg (2005)

16. Clark, K.G., Feigenbaum, L., Torres, E.: Serializing sparql query results in json W3C Working Group Note 18 June 2007 (2007) 